\title{
Immediate early response protein 2 promotes the migration and invasion of hepatocellular carcinoma cells via regulating the activity of Rho GTPases
}

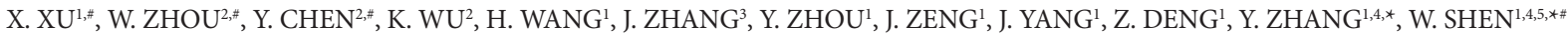 \\ ${ }^{1}$ Department of Cell Biology, School of Medicine, Yangzhou University, Yangzhou, China; ${ }^{2}$ Department of Internal Medicine, Affiliated Hospital \\ of Yangzhou University, Yangzhou, China; ${ }^{3}$ Department of Internal Medicine, Northern Jiangsu People's Hospital Affiliated to Yangzhou Uni- \\ versity, Yangzhou, China; ${ }^{4}$ Jiangsu Co-Innovation Center for Prevention and Control of Important Animal Infectious Diseases and Zoonosis, \\ Yangzhou University, Yangzhou, China; ${ }^{5}$ Jiangsu Key Laboratory of Integrated Traditional Chinese and Western Medicine for Prevention and \\ Treatment of Senile Diseases, Yangzhou University, Yangzhou, China
}

*Correspondence: shenweigan@hotmail.com; yzzy10182001@aliyun.com

${ }^{*}$ Contributed equally to this work.

Received August 18, 2019 / Accepted October 8, 2019

\begin{abstract}
Human immediate early response 2 (IER2) has been implicated in tumor cell motility and metastasis; however, the underlying mechanisms in hepatocellular carcinoma (HCC) metastasis remain to be clarified. In this study, we demonstrate that dysregulation of IER2 was shown in HCC clinical samples, and IER2 expression resulted in the promotion of cell migration and invasion in vitro, and HCC tumor growth and pulmonary metastasis in vivo. Moreover, we showed that IER2 expression altered assembly of the actin cytoskeleton rearrangement. Furthermore, MAPK and PI3K/Akt signaling pathways induced by IER2 were confirmed to be probably involved in regulating the activity of Rho GTPases, such as RhoA, Rac1 and Cdc42. Collectively, our results indicated a significant role of IER2 in the HCC cell motility and metastasis through MAPK and PI3K/Akt signaling pathways to regulate the activity of Rho GTPases, thereby modulating actin cytoskeleton rearrangement, unveiling a novel mechanism of cell motility regulation induced by IER2.
\end{abstract}

Key words: immediate early response 2, hepatocellular carcinoma cell, Rho GTPase, cell migration and invasion

Immediate early response 2 (IER2) is identified as a member of the immediate early response gene family, which is an important regulator of cellular response to stimuli such as stress and hormones [1-10]. Human IER2 encodes a 223-amino acid protein, which is a proline-, serine- and arginine-rich protein, and shares only 76\% homology with the protein encoded by pip 92 , a homologous gene in mouse [2]. Evidence is accumulating that IER2 is a DNA-binding protein which seems to act as a transcriptional factor [6], and is involved in various kinds of physiology, such as angiogenesis and cancer [11-12], although the mechanism involved is only partly understood.

Hepatocellular carcinoma (HCC) is the fifth most prevalent cancer and the third leading cause of cancer-related deaths worldwide, and the vast majority of HCC-related deaths are caused by metastasis [13-15]. Thus, understanding and elucidating the cellular mechanisms that modulate metastasis is vital to the development of effective HCC therapies. Accumulating evidence has shown that the interaction between integrin and
Rho-family GTPases (Rho GTPases), such as RhoA, Rac1 and Cdc42, regulates the motility of various cancer cells [16], and that Rho GTPases are molecular switches that regulate actin cytoskeleton reorganization and further modulate a variety of dynamic cellular functions including cell migration and invasion [17]. We have previously demonstrated that IER2 regulates $\mathrm{HCC}$ cell adhesion and motility via integrin $\beta 1$ (ITGB1)-mediated signaling pathway [18]. Although IER2 has been shown to play critical roles in tumor cell motility and metastasis and predicts poor survival of colorectal cancer patients $[12,18]$, nothing is known regarding its involvement in integrin/Rho GTPases signaling pathway.

In the present study, we demonstrated that dysregulation of IER2 was shown in HCC tissues. We revealed a crucial role for dysregulation of IER2 in regulating cell migration and invasion in vitro and in HCC growth and metastasis in vivo. Furthermore, we demonstrated a significant role of IER2 in the HCC cell motility and metastasis through activation of Rho GTPases. 


\section{Materials and methods}

Cell lines and culture conditions. Human embryonic kidney (HEK) 293T cells, purchased from the Cell Bank of the Chinese Academy of Sciences (Shanghai, China), and MHCC97H cells, obtained from the KeyGen Biotech (Nanjing, China), were cultured in Dulbecco's modified Eagle's medium (DMEM, Gibco-BRL, Grand Island, NY, USA) supplemented with $10 \%$ fetal bovine serum (FBS), 100 $\mu \mathrm{g} / \mathrm{ml}$ streptomycin and $100 \mathrm{U} / \mathrm{ml}$ penicillin, and maintained at $37^{\circ} \mathrm{C}$ in a humidified atmosphere of $5 \% \mathrm{CO}_{2}$.

Tissue specimens and clinical samples. Tissue microarray (TMA) chips which contain 75 cases of the HCC tissues and matched adjacent non-tumor liver tissues were obtained from Shanghai Outdo Biotech Company (Shanghai, China). Thirty matched fresh HCC tissues and matched adjacent non-tumor liver tissues were collected from patients with pathologically and clinically confirmed HCC at Yangzhou University Clinical Medical College. All of the patients have given informed consent. Dissected samples were frozen immediately after surgery and stored at $-80^{\circ} \mathrm{C}$. The present study was approved by the Ethics Review Committee of Yangzhou University School of Medicine and was performed in accordance with the Declaration of Helsinki. The TMA chips were selected for immunohistochemistry staining of IER2, and the HCC fresh tissues with matched adjacent non-tumor liver specimens were used for real-time reverse transcription quantitative PCR (RT-qPCR) and western blot analysis.

Immunohistochemistry. Immunohistochemistry staining of paraffin sections on the TMA chips for IER2 was carried out using UltraVision HP IHC detection kit obtained from Shanghai Universal Biotech (Shanghai, China) according to the manufacturer's instructions. Briefly, TMA chips were deparaffinized and rehydrated, and endogenous peroxidases were inactivated with $3 \%$ hydrogen peroxide in PBS. After antigen retrieval, the slides were incubated with the primary antibody at $4{ }^{\circ} \mathrm{C}$ overnight, followed by the incubation with the secondary antibody at room temperature for $1 \mathrm{~h}$, and counterstained with hematoxylin. Semiquantitative scores were used to analyze immunostaining of each HCC case in TMA, and intensity of staining was categorized into $-;+;++$; or +++ ; denoting negative, weak, moderate, or strong positive staining, respectively.

Lentiviral production and infection. The lentiviral vectors encoding IER2, shRNA against IER2, and indicated control lentiviruses were generated, and the stable cell lines expressing control (Ctrl1), IER2 (OE IER2), control-shRNA (Ctrl2), or shRNA against IER2 (KD IER2) were established and verified by RT-qPCR and western blotting as previously described [18].

RT-qPCR. Total RNA was isolated from the tissues and the cells with RNA Isolator Total RNA Extraction Reagent (VAZYME, Nanjing, China), and reverse-transcribed using HiScript 1st Strand cDNA Synthesis Kit (VAZYME,
Nanjing, China) following the manufacturer's instructions. The cDNA was subjected to real-time PCR using the SYBR Green qPCR system (Takara, Dalian, China) according to the manufacturer's instruction with the following primers: IER2 forward 5'-CCAAAGTCAGCCGCAAACGA-3' and reverse 5'-TTTCTTCCAGACGGGCTTTCTTGC-3'; and glyceraldehyde-3-phosphate dehydrogenase (GAPDH) forward 5'-GCACCGTCAAGGCTGAGAAC-3' and reverse 5'-TGGTGAAGACGCCAGTGGA-3'. GAPDH served as an internal control. The relative expression of the mRNA normalized against GAPDH was calculated using the $2^{-\triangle \Delta C T}$ methods. All experiments were performed in triplicate and repeated three times.

Western blotting. Western blotting was performed according to the standard methods. Briefly, cell lysates resolved with sodium dodecyl sulfate polyacrylamide gel electrophoresis (SDS-PAGE), and gels were transferred onto polyvinylidene fluoride membranes (Millipore, Billerica, USA) followed by blotting with primary antibodies. After incubation with the horseradish peroxidase (HRP)-conjugated secondary antibodies for $2 \mathrm{~h}$ at room temperature, protein visualization was detected with the Pierce ECL Plus western blotting substrate (Thermo Fisher Scientific, Inc.), and the GAPDH or actin was employed as an internal loading control. The following antibodies were used in the present study: mouse anti-IER2 purchased from Abcam (Cat. no. ab168980); rabbit anti-ERK1/2 (Cat. no. 4695), rabbit anti-Phospho-ERK1/2 (pERK, Cat. no. 4370), rabbit anti-p38 (Cat. no. 8690), rabbit anti-Phospho-p38 (pp38, Cat. no. 4511), rabbit anti-SAPK/JNK (JNK, Cat. no. 9252), rabbit anti-Phospho-SAPK/JNK (pJNK, Cat. no. 4668), rabbit antiactin (Cat. no. 4970), and HRP-conjugated anti-mouse IgG (Cat. no. 7076) and anti-rabbit IgG (Cat. no. 7074) purchased from Cell Signaling Technology (Danvers, MA, USA); and mouse anti-GAPDH purchased from KangChen Bio-tech (Cat. no. KC-5G4, Shanghai, China).

Cell migration and invasion assay. For wound healing assay, cells were seeded onto 6-well culture plates, serumstarved overnight and grown to confluence. Wounds were made by scraping across the cell monolayer with sterile P200 pipette tips in the middle of each well. After removing the cell debris, the remaining cells were maintained in fresh DMEM supplemented with $1 \% \mathrm{FBS}$ at $37^{\circ} \mathrm{C}$ for $24 \mathrm{~h}$. Wound closure was observed and photographed under an inverted microscope, and relative migration of cells was calculated. Transwell cell migration and invasion assays were performed as described previously [18]. In brief, cells were serumstarved overnight and then $2.5 \times 10^{4}$ cells were seeded in uncoated or Matrigel (BD Biosciences)-coated membrane of inserts with $8.0 \mathrm{~mm}$ pores $(6.5 \mathrm{~mm}$, Corning, NY, USA) of the 24-well transwell. Then the inserts were placed into the lower chamber filled with $600 \mu \mathrm{l}$ of fresh DMEM supplemented with $10 \% \mathrm{FBS}$ followed by an incubation at $37^{\circ} \mathrm{C}$ for $24 \mathrm{~h}$. The migrated or invaded cells on the lower surface of the inserts were fixed with methanol for $15 \mathrm{~min}$, and stained 
with $0.1 \%$ crystal violet for $20 \mathrm{~min}$, and counted from six random fields at $200 \times$ magnification per insert from triplicate wells. All experiments were performed in triplicate and repeated three times.

Immunofluorescence analysis. The cells seeded on sterile cover-slips in 24-well plates were fixed with $4 \%$ paraformaldehyde, permeabilized with $0.1 \%$ TritonX-100, blocked with Image-iT FX Signal Enhancer (Invitrogen), and then incubated with rhodamine-conjugated phalloidin (SigmaAldrich, USA) for $1 \mathrm{~h}$ at room temperature. After washing with PBST, the nuclei were counterstained with $0.5 \mu \mathrm{g} / \mathrm{ml}$ DAPI (Sigma-Aldrich, USA). Images were captured using a fluorescence microscope.

Rho GTPase activity assays. Rho GTPases activity was determined using the RhoA/Rac1/Cdc42 G-LISA Activation Assay Kits (Cytoskeleton Inc., Denver, CO, USA) according to the manufacturer's instructions. In brief, cells were grown to $70-80 \%$ confluence and lysed. Equalized protein extractions were distributed to a pre-coated plate, and then incubated with the antibodies specific to RhoA, Rac1, or CDC42, followed by an incubation with a HRP-conjugated secondary antibody. Active GTP-bound RhoA, Rac1, or CDC42 were detected with HRP detection reagent, and the absorbance was measured at $490 \mathrm{~nm}$ by using a microplate reader.

In vivo tumor growth and metastasis. Athymic nude mice (BALB/C-nu/nu, 6-8 weeks old, male) were obtained from the Animal Centre of Yangzhou University and maintained in a pathogen-free environment. All animal procedures were performed in compliance of the institutional ethical requirements and were approved by the Yangzhou University School of Medicine Committee for the Use and Care of Animals. For the subcutaneous inoculation models, MHCC97H cells $\left(1 \times 10^{6}\right.$ cells per mouse) were suspended in $100 \mu \mathrm{PBS}$ and then inoculated subcutaneously into the lateral flanks of nude mice. Tumor growth was monitored with calipers every five days to estimate the volume, and the tumor volume was calculated using the formula: Volume $\left(\mathrm{mm}^{3}\right)=\left[\right.$ width ${ }^{2} \times$ length]/2. For the tail vein metastasis models, $1 \times 10^{6}$ cells suspended in $100 \mu \mathrm{l}$ PBS were injected into tail veins of nude mice. All of the mice were sacrificed under anesthesia at six weeks for tumor growth assay or 24 days for tail vein metastasis assay after the inoculation. Tumors and lung tissues were removed, fixed in formalin, and embedded in paraffin. Consecutive sections were analyzed by hematoxylin and eosin (H\&E) staining.

Statistical analysis. The Student's t-test, one-way ANOVA, and Mann-Whitney $U$ test were performed to analyze in vitro and in vivo data by software SPSS 17.0, and p-values $<0.05$ were considered statistical significant.

\section{Results}

Analysis of the expression of IER2 in HCC tissues. We first analyzed the mRNA level of IER2 in clinical specimens from the TCGA database (https://genome-cancer. ucsc.edu). We found that IER2 mRNA is downregulated in HCC tumor tissues compared with matched non-tumor liver tissues (Figure 1A). To validate the analysis finding, we examined the expression of IER2 in 30 pairs of human HCC clinical samples and their corresponding paracancerous tissues by RT-qPCR and western blotting. We found that the expression of IER2 was significantly reduced in 26/30 HCC samples compared with that in matched paracancerous tissues (Figures 1B, 1C). We also examined the expression of IER2 in 75 pairs of clinical HCC samples by immunohistochemistry. Results from TMA analysis showed that there were 72 cases of positive expression and 9 cases of strong positive expression of IER2 in 75 HCC tissues, whereas in the corresponding paracancerous tissues, there were 74 cases of positive expression and 15 cases of strong positive expression of IER2 (Figures 1D, 1E). Furthermore, we have previously performed western blotting analysis to evaluate the expression of IER2 in a panel of HCC cell lines HepG2, SMMC7721, SK-HEP1 and MHCC97H cells (four HCC cell lines with increasing spontaneous metastatic potential), and we demonstrated that higher level of IER2 was shown in SK-HEP1 and MHCC97H cells, which have high metastatic potential, and lower level of IER2 was observed in HepG2 and SMMC7721 cells, which have low metastatic potential [18], suggesting that IER2 expression was positively correlated with the metastatic potential of the HCC cell lines. Therefore, we speculate that IER2 may serve as a regulator in HCC.

IER2 regulated tumor growth and metastasis. To explore whether IER2 played a role in tumor growth and metastasis in vivo, stably overexpressing and knocking down IER2 in MHCC97H cells were established and verified as previously described [18]. MHCC97H cells stably overexpressing IER2, knocking down IER2 and their corresponding control cells were subcutaneously injected into nude mice, respectively. Tumor bearing mice were sacrificed and the tumors were dissected at the end of the experiment. Compared to the indicated control group, injection of mice with $\mathrm{MHCC} 97 \mathrm{H}$ cells stably overexpressing or knocking down IER2 resulted in an obvious increase of tumor size and weight, especially in the group of cells overexpressing IER2 (Figures 2A-2C). Immunohistochemical staining and RT-qPCR verified the IER2 overexpression and knockdown in the indicated tumor tissues (Figures 2D, 2E). We also examined the functional role of IER2 in vivo in tumor metastasis using the tail vein metastasis models. MHCC97H cells stably overexpressing IER2, knocking down IER2 and their corresponding control cells were injected into the tail veins of nude mice, respectively. Twenty-four days later, nude mice were sacrificed and the tumor metastases were analyzed. We found that the number of pulmonary nodules was significantly increased in mice injected with MHCC97H cells stably overexpressing IER2, compared to those injected with control (Figure 2F). $\mathrm{H} \& \mathrm{E}$ staining verified the observed pulmonary nodules in overexpressing IER2 cells, some metastatic lesions were 


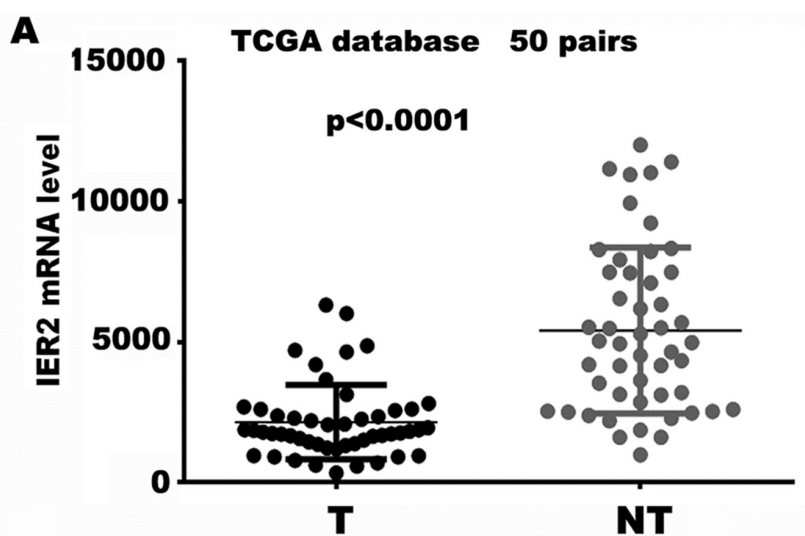

C

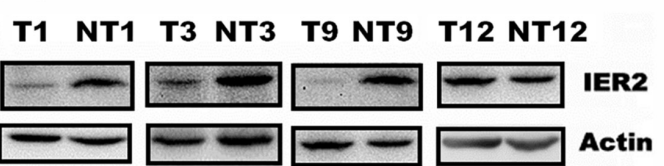

$\mathbf{E}$

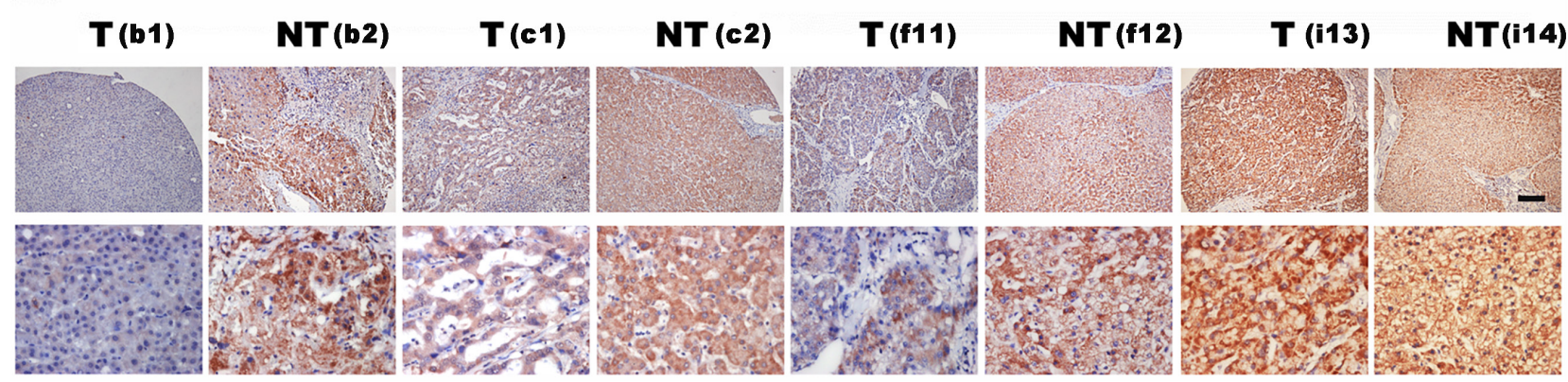

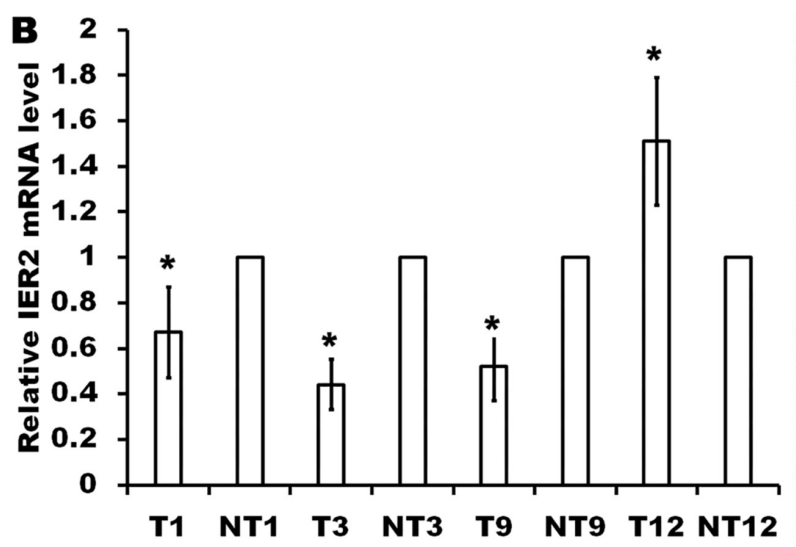

$\begin{array}{lllllllll}\text { T1 } & \text { NT1 } & \text { T3 } & \text { NT3 } & \text { T9 } & \text { NT9 } & \text { T12 NT12 }\end{array}$

Figure 1. Expression of IER2 in HCC tissues. A) Analysis of IER2 mRNA levels in 50 pairs of HCC tissues and matched non-tumor liver tissues from the TCGA database. B) IER 2 mRNA levels were measured with RT-qPCR in 30 pairs of human HCC clinical samples and their corresponding paracancerous tissues. Data were representative of four pairs. ${ }^{\star} \mathbf{p}<0.05$ vs. the matched paracancerous tissues. C) IER2 protein levels were determined by western blotting in 30 pairs of human HCC clinical samples and their corresponding paracancerous tissues. Actin protein served as a loading control, and representative blots were shown from four pairs. D and E) IER2 expression in TMA chips including 75 cases of the HCC tissues and matched adjacent non-tumor liver tissues was detected by immunohistochemistry. Representative images of the 4 pairs with different semiquantitative scores of immunostaining of IER 2 were shown and the number of the 4 pair of HCC samples from TMA chips were labelled. Scale bar; $100 \mu \mathrm{m}$.

found in lung tissue compared with the control group (Figure 2G). Taken together, these results indicated that IER2 could regulate HCC growth and metastasis in vivo.

IER2 modulated the migration and invasion of HCC cells through regulating actin cytoskeleton rearrangement. In order to verify whether IER2 regulated the metastasis of HCC by influencing cell motility, we first examined IER2 functions in cell migration and invasiveness in vitro. MHCC97H cells with IER2 overexpression showed rapid wound closure in wound-healing assays, and their migration and invasion efficiency obviously increased in transwell assays compared with corresponding control cells, whereas knockdown of IER2 resulted in a reduction of the cell motility (Figure 3). Accordingly, we proposed that IER2 played a role in HCC cell invasiveness and metastasis. Considering that cellular motility is closely coupled with the protru- 

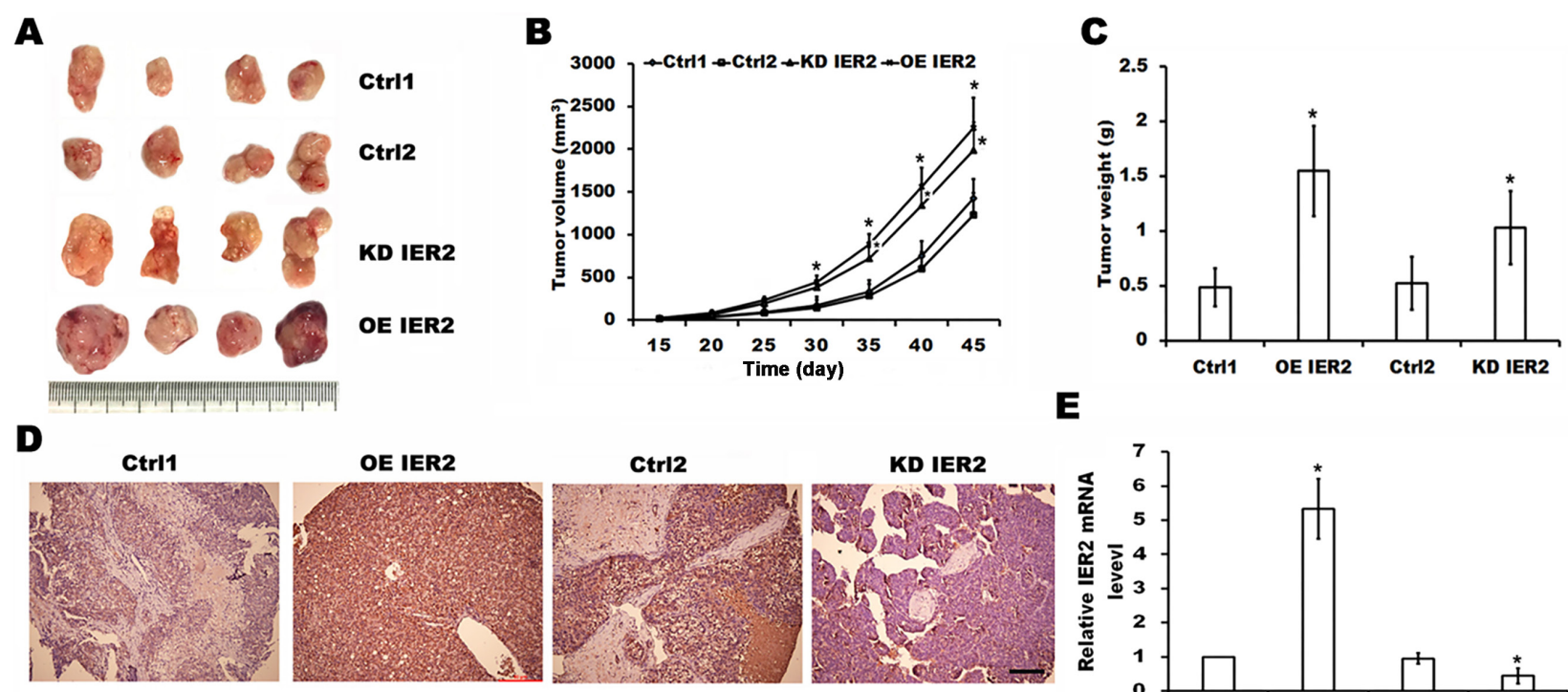

$\mathbf{E}$
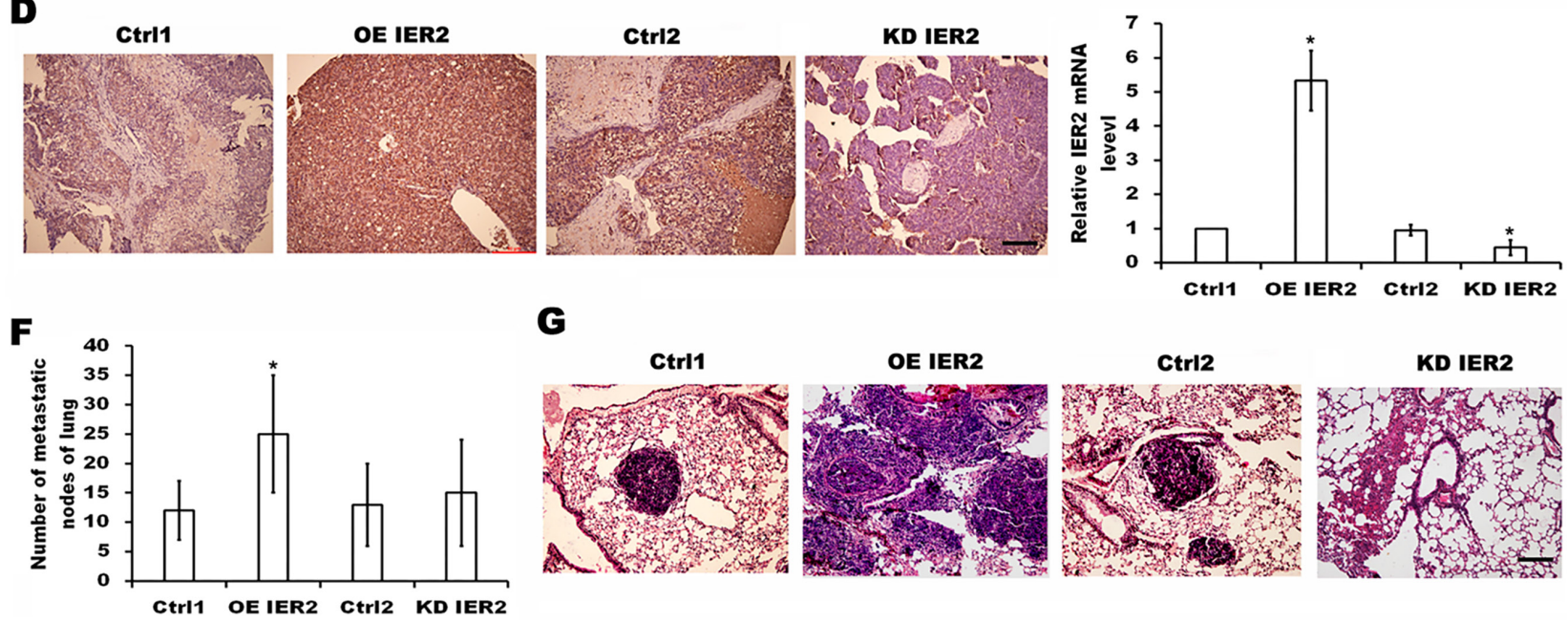

Figure 2. IER2 regulated tumor growth and metastasis. A-C) Representative the subcutaneous inoculation models. MHCC97H cells stably over-expressing IER2, knocking down IER2 and their corresponding control cells were subcutaneously injected into nude mice. Representative images of the different groups were shown at six weeks following subcutaneous inoculation (A), tumor volume (B) and tumor weight (C) were analyzed. Data were shown as the mean $\pm \mathrm{SD},{ }^{*} \mathbf{p}<0.05$, versus their corresponding control (Ctrl). D) Representative immunohistochemical staining IER2 in tumor tissues of different groups. Scale bar; $100 \mathrm{~mm}$. E) The expressions of IER2 were detected by RT-qPCR in the indicated tumor tissues. GAPDH was used for normalization. ${ }^{*} \mathbf{p}<\mathbf{0 . 0 5}$, versus their corresponding control (Ctrl). F and G) Representative the tail vein metastasis models. Number of lung metastatic foci in each group were analyzed (F). Data were shown as the mean $\pm \mathrm{SD},{ }^{\star} \mathbf{p}<0.05$, versus the Ctrl1. G) Representative H\&E staining images of lung tissues from different groups. Scale bar; $100 \mu \mathrm{m}$.

members of the Rho family of small GTPases, such as RhoA, $\mathrm{Rac1}$ and Cdc42, as key regulators of the actin cytoskeletal rearrangement in the process of tumor cell migration, invasion and metastasis [20]. To examine whether IER2-induced actin reorganization is associated with the Rho GTPases, we then determined the activity of RhoA, Rac1 and Cdc42 in MHCC97H cells by using the RhoA/ Rac1/Cdc42 G-LISA Activation Assay. Compared with those in the indicated control cells, overexpression of IER2 enhanced the activity of both Racl and Cdc42 but reduced RhoA activity, and thereby promoted lamellipodia formation and cell migration, whereas IER2 knockdown showed a significant reduction of the activity of Racl and a slight but non-significant alteration of the activity of RhoA and Cdc42 in MHCC97H cells (Figures 4B-4D). These results clearly indicated that IER2 modulates the activity of the Rho family of GTPases, which might be the major cause of IER2 in lamellipodia formation of motile protrusions, thus further control the migration and invasion of MHCC97H cells.
IER2 enhanced cell motility by activating the downstream signaling molecules. We have previously demonstrated that IER2 regulates HCC cell adhesion and motility via ITGB1-mediated signaling pathway [18], which may trigger several downstream signaling pathways such as PI3K-Akt and MAPK (ERK, JNK and p38) to regulate the activity of the Rho family of GTPases, resulting in reorganization of the actin cytoskeleton [21-22]. Thus, we further performed western blotting to explore whether IER2 expression regulated the PI3K/Akt and MAPK signaling pathways. As shown in Figure 5, the phosphorylation levels of p38 (pp38), ERK (p-ERK) and Akt (p-Akt) were increased in IER2 overexpression cells compared to control cells, while IER2 knockdown resulted in upregulation of pp38 but downregulation of p-ERK. Neither overexpression nor knockdown of IER2 had an obvious effect on the expression of Akt, ERK, p38, JNK and the phosphorylation of JNK (p-JNK). These results suggested that IER2-induced the activation of Rho GTPases (RhoA 
$\mathbf{A}$
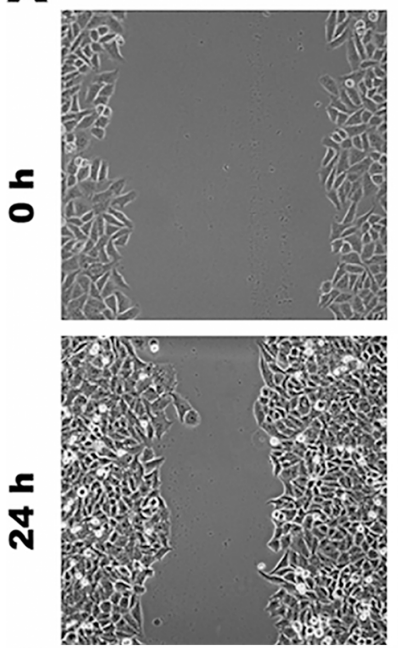

$\mathbf{B}$
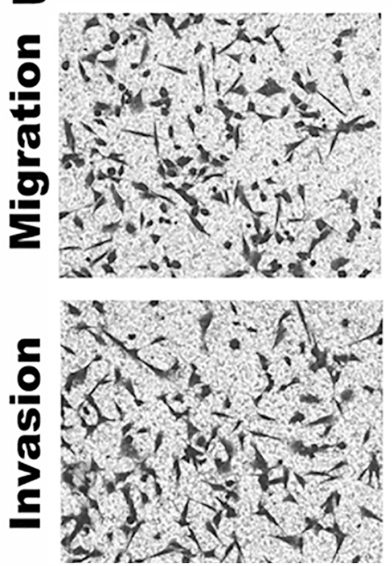

C

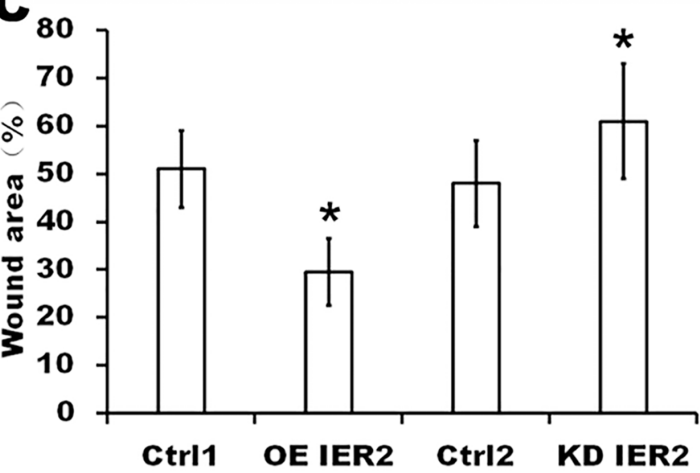

OE IER2

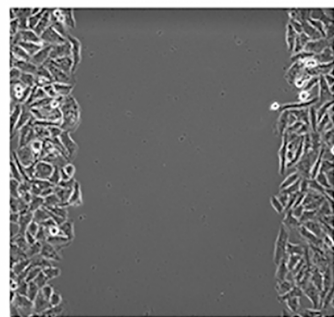

OE IER2
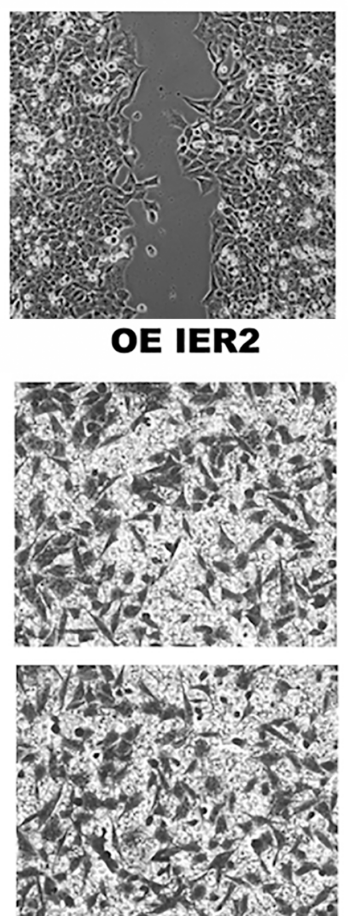

D

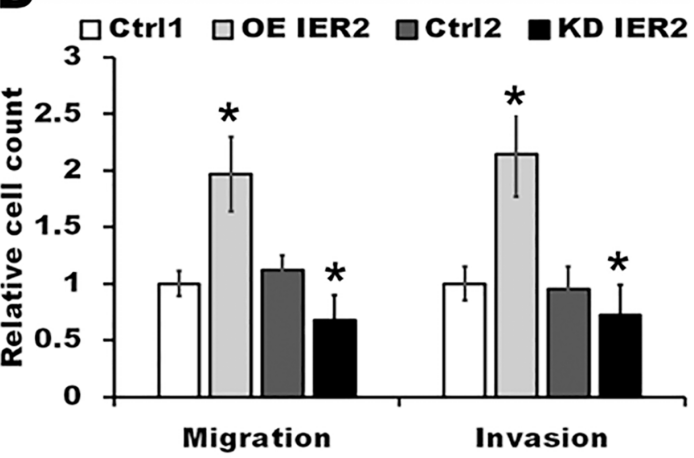

Figure 3. Effect of IER2 overexpression or knockout on migration and invasion of MHCC97H cells. A) Representative the wound healing assay to assess the migration of IER2-overexpressing or -silenced MHCC97H cells (magnification, $\times 100$ ). B) Representative transwell cell migration and invasion assays in IER2-overexpressing or -silenced MHCC97H cells (magnification, $\times 200)$. Quantification of relative wound healing (C) and numbers of migrated and invaded cells (D) was shown. Data were shown as mean $\pm \mathrm{SD}$. ${ }^{*} \mathrm{p}<0.05$, versus the indicated control (Ctrl).

Rac1 and Cdc42) in MHCC97H cells might be mediated, at least in part, by regulating the MAPK (ERK and p38) and Akt pathways, thereby modulating the migration and invasion of HCC cells.

\section{Discussion}

HCC is a poor prognosis tumor with a high frequency of relapse and metastasis, which is a complex cascade of events, 
A
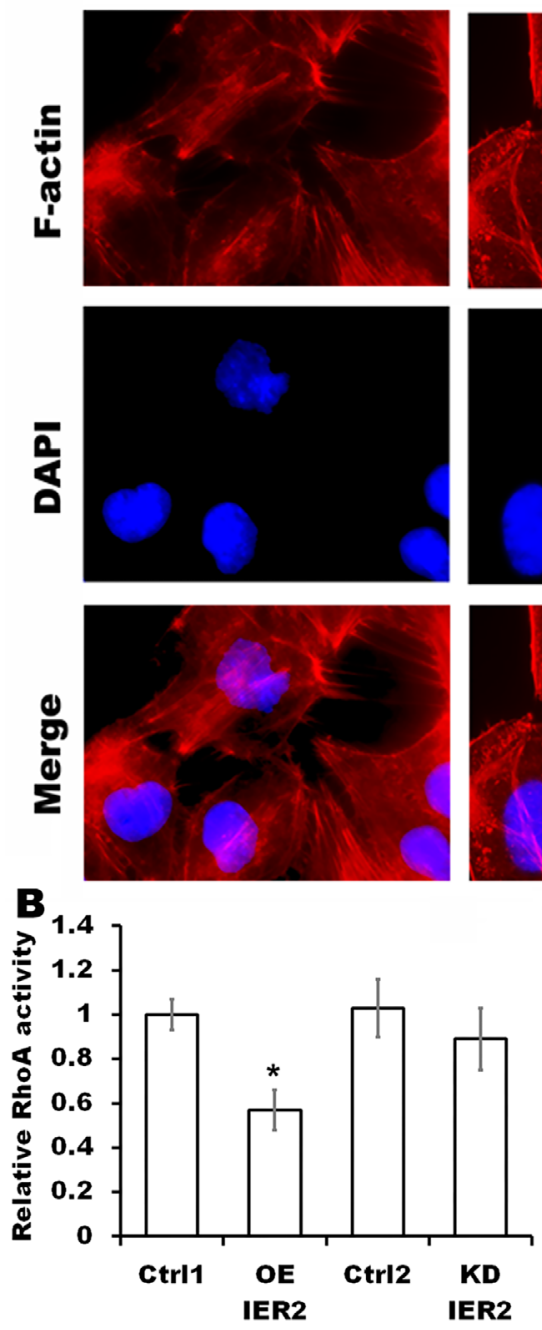

OE IER2
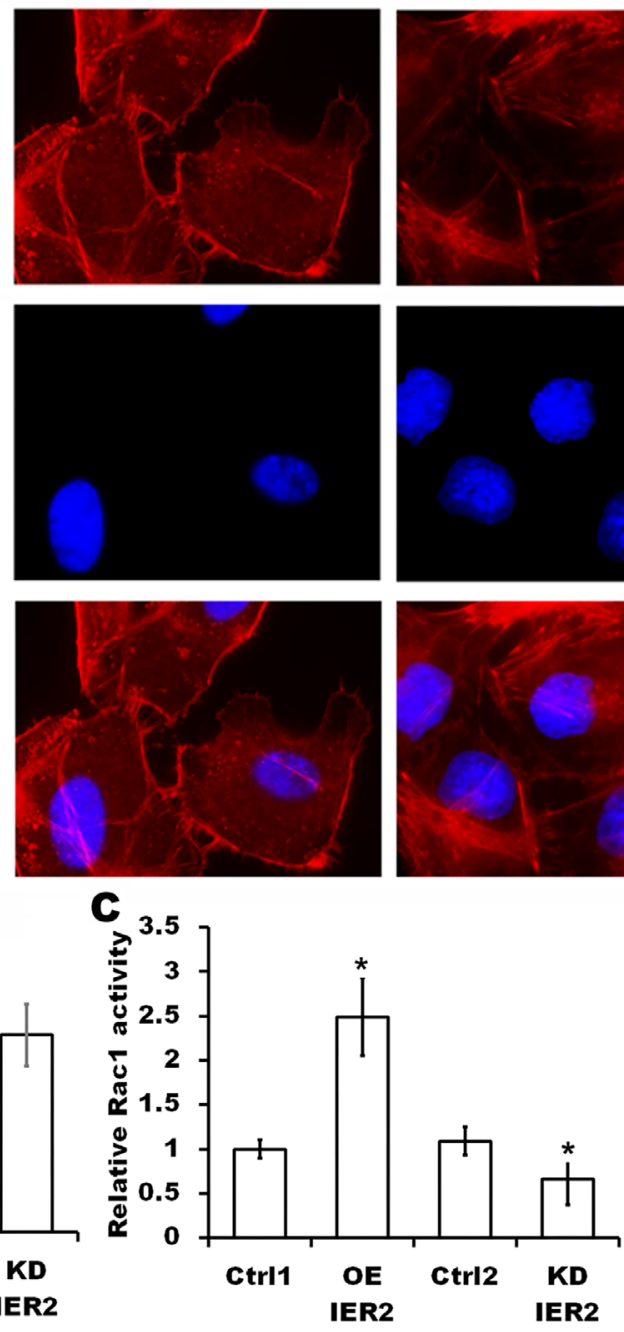

Ctrl2
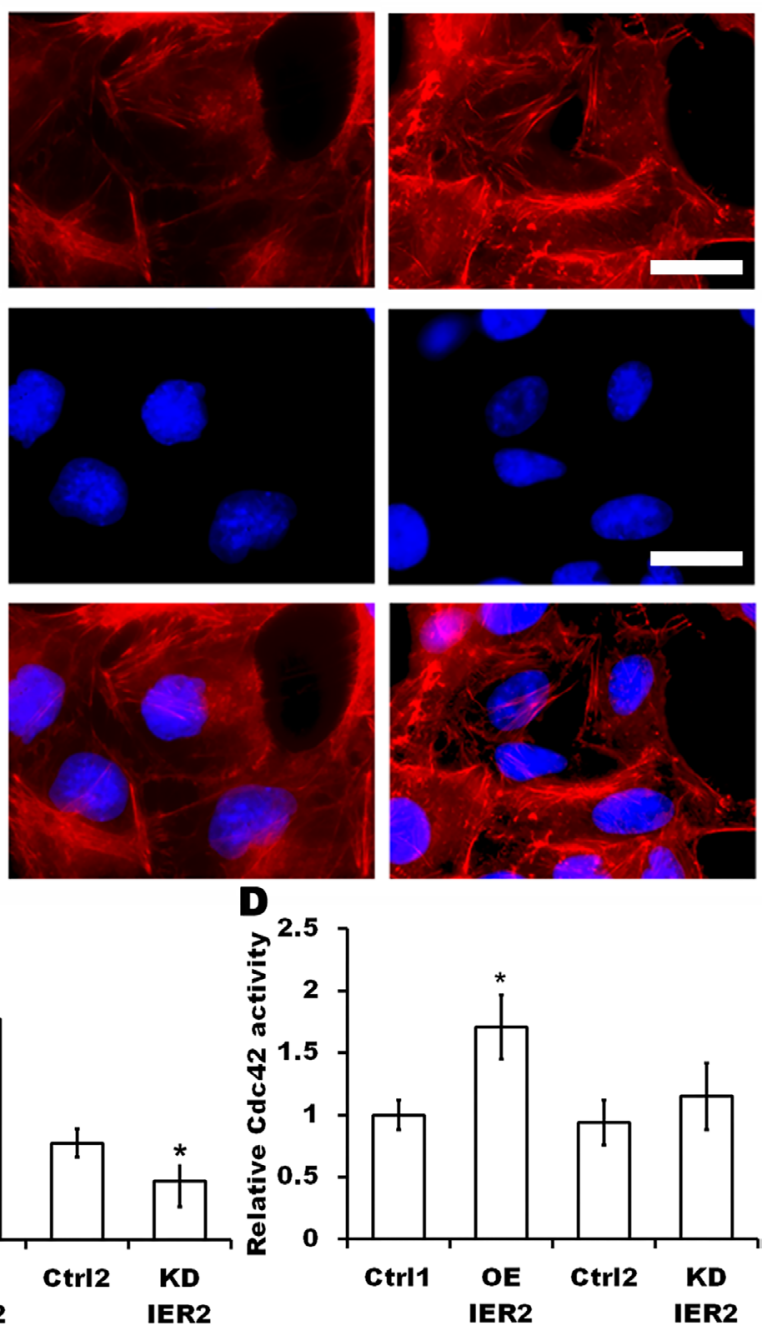

Figure 4. IER2-induced actin reorganization was associated with the Rho GTPases. A) Representative images of F-actin (red) and DAPI (blue) staining. Scale bar; $20 \mu \mathrm{m}$. The images represent the cells from three independent experiments. B-D) The activity of RhoA, Racl and Cdc42 was determined by G-LISA Activation Assay Kit. Data were shown as mean \pm SD of three independent experiments. ${ }^{*}$ p $<0.05$, versus the indicated control $($ Ctrl).

including the migration and invasion of HCC cells. Therefore, better knowledge about the mechanisms that regulate the processes of migration and invasion is essential for the development of diagnostic markers and effective approaches to decrease HCC mortality. There is evidence showing that IER2 has some DNA binding ability and is involved in tumor cell motility and metastasis $[6,12,18]$, however, the underlying mechanisms in HCC metastasis remain elusive. In this study, we provided evidence that dysregulation of IER2 was shown in HCC clinical samples, and revealed a crucial role for dysregulation of IER2 in regulating cell migration and invasion in vitro and in HCC growth and metastasis in vivo. Furthermore, we demonstrated a significant role of IER2 in the HCC cell motility and metastasis through the activation of the Rho family of GTPases, suggesting that IER2 may function as an important regulator in HCC metastasis.
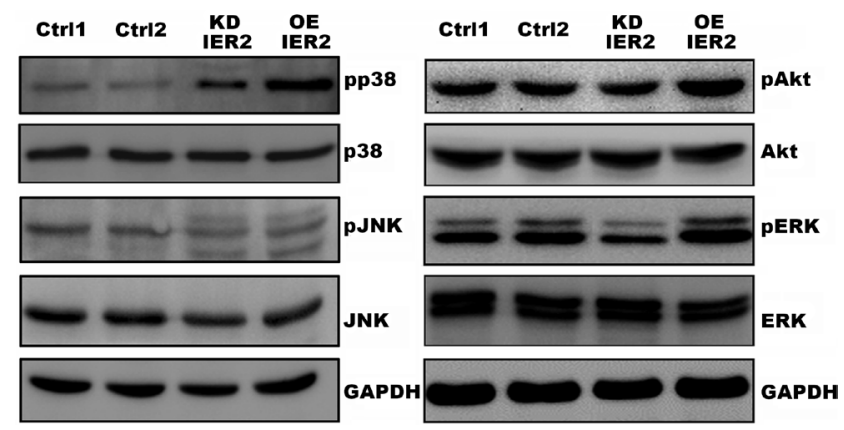

Figure 5. Effect of IER2 on expression of Akt, ERK, JNK and p38 in MHCC97H cells. Protein levels of Akt, pAkt, ERK, pERK, JNK, pJNK, p38 and pp38 were analyzed from MHCC $97 \mathrm{H}$ cells stably over-expressing IER2, knocking down IER2 or their corresponding control cells by western blotting with the corresponding antibodies, and GAPDH was served as loading control, and three separate experiments were performed. 
Although IER2 has been described to play roles in tumor cell motility and metastasis $[12,18]$, and our previous study showed that the elevated expression of IER2 was positively correlated with the high metastatic potential of certain HCC cells [18], IER2 initially exhibited dysregulation in the HCC tissues in the present study. As enhanced tumor cell motility always promotes cell metastasis, we first evaluated whether dysregulation of IER 2 could also induce HCC growth and metastasis in vivo. We demonstrated that ectopic IER2 expression in MHCC97H cells promoted HCC tumor growth and metastasis in mice in vivo, whereas silencing IER2 expression in MHCC97H cells promoted HCC tumor growth but displayed non-significant alteration of tumor metastasis in mice. Moreover, we also demonstrated that IER2 overexpression acquired an obviously increased ability for migration and invasion, underwent rearrangement of the actin cytoskeleton. Thus, these findings provide evidence supporting the pro-metastatic function of IER2 in HCC.

The Rho family of GTPases, including RhoA, Racl and Cdc42, are important regulators of actin cytoskeleton dynamics that potentiate cell migration and invasion [20-24]. Generally, Racl and Cdc42 act at the leading edge of the cell to induce the formation of lamellipodia and cell polarization, respectively, to promote cell migration, whereas RhoA acts in the cell body to facilitate contraction [23-25]. In this study, we demonstrated that overexpression of IER2 increased the activity of both Racl and Cdc42 but reduced the activation of RhoA in MHCC97H cells, and thereby contributed to the formation of lamellipodia, leading to the promotion of cell migration and invasion. Interestingly, we also found a correlation between the depletion of IER 2 and Rac1 activity but not Cdc42 and RhoA activity in MHCC97H cells. Based on this evidence, we assumed that IER2 might be involved in regulating actin dynamics through Rho family GTPases and could thereby affect HCC motility.

Our previous study showed that IER2 increased HCC cell adhesion and motility through transcriptional regulation of ITGB1 and subsequently activating the ITGB1FAK-Src-paxillin signal pathway [18]. As integrin-mediated signaling pathway may also function as critical mediators of the PI3K-Akt and MAPK signaling pathways to regulate the activation of Rho family of GTPases [21, 22, 26], this prompted us to investigate whether IER2 can further modulate the downstream signaling events involving PI3K-Akt and MAPK (ERK, JNK and p38) pathways. In the present study, we demonstrated that IER2 overexpression significantly upregulated the levels of the phosphorylation of p38, ERK and Akt, while IER2 knockdown resulted in the downregulation of the phosphorylation of ERK but upregulation of the phosphorylation of p38. However, the mechanism by which IER2 affects PI3K-Akt and MAPK remains to be further explored. Taken together, our observations demonstrated that IER2-induced the activation of Rho GTPases in MHCC97H cells might be mediated, at least in part, by regulating the MAPK (ERK and p38) and Akt signaling pathways, thereby regulating the migration and invasion of HCC cells.

In conclusion, we provided novel insight into the significance of IER2 expression in HCC, and revealed a crucial role of IER2 in HCC tumor growth and metastasis. Our findings showed that IER2 played an important role during HCC cell migration and invasion through MAPK and PI3K-Akt signaling pathways to regulate the activity of Rho GTPases, thereby modulating actin cytoskeleton rearrangement. Further study of the specific mechanism as to how IER2 aberrant expression impact the occurrence, malignant degree and metastasis of HCC is ongoing, however, the findings presented here may contribute to improving our understanding of IER2 function in HCC and may also be important for the development of novel HCC biomarkers.

Acknowledgments: This work was supported by the National Nature Science Foundation of China (No. 81172278 and 81672336).

\section{References}

[1] SHIMIZU N, OHTA M, FUJIWARA C, SAGARA J, MOCHIZUKI $\mathrm{N}$ et al. Expression of a novel immediate early gene during 12-O-tetradecanoylphorbol-13-acetate-induced macrophagic differentiation of HL-60 cells. J Biol Chem 1991; 266: 12157-12161.

[2] CHEN L, MA S, LI B, FINK T, ZACHAR V et al. Transcriptional activation of immediate-early gene ETR101 by human T-cell leukaemia virus type I Tax. J Gen Virol 2003; 84: $3203-$ 3214. https://doi.org/10.1099/vir.0.19283-0

[3] DENG YJ, HUANG ZX, ZHOU CJ, WANG JW, YOU Y et al. Gene profiling involved in immature CD4+T lympho $\neg$ cyte responsible for systemic lupus erythematosus. Mol Immunol 2006; 43: 1497-1507. https://doi.org/10.1016/j.molimm.2005.07.039

[4] SHEN QY, ZHENG SS. Identification of genes differentially expressed in monocyte-derived dendritic cells with 1 alpha, 25-dihydroxyvitamin D3 using cDNA arrays. J Zhejiang Univ Sci 2004; 5: 222-225. https://doi.org/10.1007/bf02840927

[5] ZENG F, HON CC, SIT WH, CHOW KY, HUI RK et al. Molecular characterization of Coriolus versicolor PSP-induced apoptosis in human promyelotic leukemic HL-60 cells using cDNA microarray. Int J Oncol 2005; 27: 513-523.

[6] TAKAYA T, KASATANI K, NOGUCHI S, NIKAWA J. Functional analyses of immediate early gene ETR101 expressed in yeast. Biosci Biotechnol Biochem 2009; 73: 1653-1660. https://doi.org/10.1271/bbb.90162

[7] HESS S, RHEINHEIMER C, TIDOW F, BARTLING G, KAPS C et al. The preprogrammed host: Chlamydia trachomatis-induced up-regulation of glycoprotein 130 cytokines, transcription factors, and antiapoptotic genes. Arthritis Rheum 2001; 44: 2392-2401. https://doi.org/10.1002/15290131(200110)44:10<2392::aid-art404>3.0.co;2-i

[8] HONG SK, DAWID IB. FGF-dependent left-right asymmetry patterning in zebrafish is mediated by Ier2 and Fibp1. Proc Natl Acad Sci U S A 2009; 106: 2230-2235. https://doi. org/10.1073/pnas.0812880106 
[9] WONG VC. Nuclear EGFR and Integrator/Super Elongation Complex concurrently binds to Immediate Early Genes for gene transactivation. J Cancer 2018; 9: 108-116. https://doi. org/10.7150/jca.21925

[10] UEDA T, KOHAMA Y, SAKURAI H. IER family proteins are regulators of protein phosphatase $\mathrm{PP} 2 \mathrm{~A}$ and modulate the phosphorylation status of CDC25A. Cell Signal 2019; 55: 81-89. https://doi.org/10.1016/j.cellsig.2018.12.012

[11] WU WJ, ZHANG XZ, LV HN, LIAO YX, ZHANG WC et al. Identification of immediate early response protein 2 as a regulator of angiogenesis through the modulation of endothelial cell motility and adhesion. Int J Mol Med 2015; 36: 1104-1110. https://doi.org/10.3892/ijmm.2015.2310

[12] NEEB A, WALLBAUM S, NOVAC N, DUKOVIC-SCHULZE S, SCHOLL I et al. The immediate early gene Ier2 promotes tumor cell motility and metastasis, and predicts poor survival of colorectal cancer patients. Oncogene 2012; 31: 3796-3806. https://doi.org/10.1038/onc.2011.535

[13] MITTAL S, EL-SERAG HB. Epidemiology of hepatocellular carcinoma: consider the population. J Clin Gastroenterol 2013; 47: S2-6. https://doi.org/10.1097/ MCG.0b013e3182872f29

[14] FORNER A, REIG M, BRUIX J. Hepatocellular carcinoma. Lancet 2018; 391: 1301-1314. https://doi.org/10.1016/ S0140-6736(18)30010-2

[15] XU XL, LOU Y, TANG JW, TENG Y, ZHANG ZC et al. The long non-coding RNA Linc-GALH promotes hepatocellular carcinoma metastasis via epigenetically regulating Gankyrin. Cell Death Dis 2019; 10: 86. https://doi.org/10.1038/s41419019-1348-0

[16] PARRI M, CHIARUGI P. Rac and Rho GTPases in cancer cell motility control. Cell Commun Signal 2010; 8: 23. https://doi.org/10.1186/1478-811X-8-23

[17] CLEGHORN WM, BULUS N, KOOK S, GUREVICH VV, ZENT R et al. Non-visual arrestins regulate the focal adhesion formation via small GTPases RhoA and Rac1 independently of GPCRs. Cell Signal 2018; 42: 259-269. https://doi. org/10.1016/j.cellsig.2017.11.003
[18] XU ZX, ZHU L, WU WJ, LIAO YX, ZHANG WC et al. Immediate early response protein 2 regulates hepatocellular carcinoma cell adhesion and motility via integrin $\beta 1$ mediated signaling pathway. Oncol Rep 2017; 37: 259-272. https://doi.org/10.3892/or.2016.5215

[19] POLLARD TD, BORISY GG. Cellular motility driven by assembly and disassembly of actin filaments. Cell 2003; 112: 453-465. https://doi.org/10.1016/s0092-8674(03)00120-X

[20] MACHACEK M, HODGSON L, WELCH C, ELLIOTT H, PERTZ O et al. Coordination of Rho GTPase activities during cell protrusion. Nature 2009; 461: 99-103. https://doi. org/10.1038/nature08242

[21] CHOI C, KWON J, LIM S, HELFMAN DM. Integrin $\beta 1$, myosin light chain kinase and myosin IIA are required for activation of PI3K-AKT signaling following MEK inhibition in metastatic triple negative breast cancer. Oncotarget 2016; 7: 63466-63487. https://doi.org/10.18632/oncotarget. 11525

[22] NAYAK RC, CHANG KH, VAITINADIN NS, CANCELAS JA. Rho GTPases control specific cytoskeleton-dependent functions of hematopoietic stem cells. Immunol Rev 2013; 256: 255-268. https://doi.org/10.1111/imr.12119

[23] RAFTOPOUlOU M, HALL A. Cell migration: Rho GTPases lead the way. Dev Biol 2004; 265: 23-32. https://doi. org/10.1016/j.ydbio.2003.06.003

[24] RIDLEY AJ. Rho GTPases and actin dynamics in membrane protrusions and vesicle trafficking. Trends Cell Biol 2006; 16: 522-529. https://doi.org/10.1016/j.tcb.2006.08.006

[25] TZIMA E. Role of small GTPases in endothelial cytoskeletal dynamics and the shear stress response. Circ Res 2006; 98: 176-185. https://doi.org/10.1161/01. RES.0000200162.94463.d7

[26] BOLÓS V, GASENT JM, LÓPEZ-TARRUELLA S, GRANDE E. The dual kinase complex FAK-Src as a promising therapeutic target in cancer. Onco Targets Ther 2010; 3: 83-97. https://doi.org/10.2147/ott.s6909 